\title{
Do patients need a psychiatric emergency clinic?
}

\author{
Robert F. KeHOe, Registrar; and Richard Newton, Medical Research Fellow, Royal \\ Edinburgh Hospital, Morningside Terrace, Edinburgh EH10 5HF (Correspondence)
}

When planning new service initiatives it is important to review the role and functioning of existing services. As more patients are discharged from long-stay beds, there is increasing concern regarding the quality of community care and the accessibility of urgent psychiatric help. The Department of Health has recently expressed concern over the level of such services provided (DoH, 1989). In Edinburgh, alternative methods of delivering psychiatric emergency care are currently being considered. Community-orientated crisis intervention teams may be useful, but only a small proportion of people presenting to general psychiatric services fit into the stereotyped model of crisis. Centres with short-term admission facilities have been developed in other countries and within the United Kingdom many general hospital casualty departments have a psychiatrist available on call. Walk-in or self-referral psychiatric clinics provide an easily accessible service but few have been systematically reported (Lim, 1983; Haw, 1987). Such clinics have been described as seeing mainly chronic patients of the hospital who are already in current contact and who present with less severe psychiatric disorders than GP-referred cases. In a climate of NHS financial restraint it is pertinent to ask whether such clinics are needed.

This study considers the merits of such an emergency service by examining the differences between self- and GP-referred daytime cases presenting to the Royal Edinburgh Hospital (REH) Emergency Clinic. It compares the findings with published reports of other clinics and contrasts the daytime referrals with those 'out-of-hours' to the same service as described by Blaney \& West (1987).

\section{The study}

\section{The service}

The REH is situated in the centre of its catchment area, serving a population of 485,000 . The psychiatric service was sectorised in 1975. Edinburgh is a compact city and the hospital is the only one in the area providing an acute adult psychiatric service. From the hospital there is a relatively low rate of emergency domiciliary visits for adults. The average waiting time for a consultant out-patient clinic is $2-3$ weeks and urgent appointments are available to GPs within 2-3 days. A 24-hour service has been available for many years, seeing GP and other agency referrals as well as self-referrals. The clinic currently sees 2,100 patient presentations per year, 900 of which are in office hours. During the day, patients are seen in the out-patient department which has reception staff and one trained nurse available. At night, patients are seen in a room off the main hospital corridor. Two part-time registrars cover the daytime clinic with two sessions being shared by a rotation of other trainees to allow for study leave. Between $5 \mathrm{pm}$ and $9 \mathrm{am}$ and at weekends the on-call registrar sees emergency referrals as well as having responsibility for inpatients. The part-time registrars have at least 2-3 years experience and receive supervision weekly from a consultant responsible for the running of the service and can receive advice from the on-call consultant.

Data were collected prospectively over four months (March-June 1989) on all cases seen by the two parttime registrars at the clinic $(75 \%$ of all cases, allowing for covered sessions and leave). During the clinical assessment of each case a simple questionnaire was completed recording basic demographic data and details of the precipitants, diagnosis and immediate management. Precipitant factors of each visit were categorised as psychiatric, social, financial or relationship difficulties and were ranked according to importance. Current and past psychiatric contact was determined by direct questioning and reference to case notes when available. Diagnoses were classed as acute if symptoms were of less than two months duration and as chronic if more than two months.

The $\chi^{2}$ test was used to determine the statistical significance of comparisons of different variables between GP and self-referral cases.

\section{Findings}

(a) Presentation

Of the 181 cases seen, $44 \%$ were self-referred. There was no significant difference in sex ratio within the total referrals, but males represented $65 \%$ of selfreferrals $\left(\chi^{2}=8.13\right.$, d.f. $\left.=1, P<0.01\right)$. Psychiatric symptoms were judged as the most common precipitant of referral. However, in 33 of the 102 GP referred cases non-psychiatric factors were 
considered to have been the precipitant of referral, the most common being relationship or social problems. Within the self-referred group, 46 of the 79 presentations were precipitated by non-psychiatric factors $\left(\chi^{2}=12.1\right.$, d.f. $\left.=1, P<0.01\right)$, the most common being alcohol intoxication (20/79), and less commonly social or relationship crises. Only $2 \%$ of cases were from beyond the hospital catchment area and $3 \%$ were of no fixed abode.

\section{(b) Diagnosis}

Of the self-referred group, $35 \%$ had chronic alcohol problems, many presenting acutely drunk. Of the GP-referred group, $20 \%$ also had alcohol problems but few were intoxicated. Affective disorders $(32 \%$ of GP- and $13 \%$ of self-referrals) and schizophrenia ( $24 \%$ of GP- and $22 \%$ of self-referrals) were the other common diagnoses, with adjustment reaction, phobic or generalised anxiety and personality disorder accounting for most of the other cases. Drugrelated problems made up only $2 \%$ of presentations. Of GP referrals, $76 \%$ presented with an acute diagnosis compared to $22 \%$ of self-referrals $\left(\chi^{2}=25.2\right.$, d.f. $=1, P<0.01)$. This difference was most apparent in those patients with a diagnosis of schizophrenia. All but $3 \%$ of the presentations were assigned a psychiatric diagnosis.

\section{(c) Previous psychiatric contact}

Twenty per cent of self-referrals and $31 \%$ of GPreferrals had had no previous psychiatric contact. Of those patients with psychiatric histories, $32 \%$ of selfreferrals and $15 \%$ of GP-referrals were in current contact with the hospital.

Of the self-referred group, $38 \%$ had been seen at the Emergency Clinic within the last year, compared to $22.5 \%$ of GP-referrals. Of all referrals, $8 \%$ had been seen on more than three occasions over the past year, by day or night.

\section{(d) Immediate management}

Of GP referrals, $51 \%$ were admitted and $27 \%$ given urgent out-patient appointments, compared to $13 \%$ and $25 \%$ respectively of self-referrals $\left(\chi^{2}=32.1\right.$, d.f. $=3, P<0.001$ ). Of the GP referrals, $22 \%$ were offered non-urgent out-patient or other agency appointments as compared to $62 \%$ of the selfreferred group.

\section{Comment}

This study is limited by its opening design and by any pre-existing bias of diagnostic decision-making. Seasonal variations were not accounted for but diagnostic categories were compared with those for other times of the year and no significant differences found.
Management of cases partly depends on local factors and may limit generalisation of findings.

GP referrals to the clinic were precipitated by psychiatric factors more often than self-referrals and more required urgent psychiatric care. Self-referrals often had pressing social and financial problems suggesting that a social-worker, if attached to the clinic, would be of benefit to such patients.

The steady staffing levels throughout the 24 hours may contribute to the similarity in the rates of admission between the office-hours and the out of hours group (as reported by Blaney \& West, 1987). Services with varying staffing levels have found varying rates of admission.

A significant number of self-referrals presented acutely drunk. Such patients are assessed as much as seems necessary; security staff have recently been employed to protect staff if required. Few people with drug-related problems were seen at the clinic. This may reflect the availability of other services within and beyond the hospital but it may be that such clients feel the Emergency Clinic has little to offer them, as registrars are, by local agreement, not empowered to prescribe the controlled drugs which they may ask for.

The high proportion of men in the self-referred group has been noted in other studies (Lim, 1983; Haw et al, 1987). GPs are more likely to refer men with psychiatric symptoms whereas many more women present with psychiatric problems to them. This may represent differences in help-seeking behaviour between the sexes, and, taken together with the emergency clinic findings, suggest that self-referral is an important pathway in seeking psychiatric help, especially for men.

Johnstone outlined the difficulties in seeking advice encountered by patients and relatives in cases of first-onset psychosis, who made many attempts to get help before they found the right agency (Johnstone et al, 1986). This problem may well apply to other diagnoses too. Much depends on local ease of access but they highlighted that an emergency clinic provides an important route of entry into psychiatric care. This is borne out by this study where $27 \%$ of all patients presenting to the clinic had no previous psychiatric contact. This conflicts with the widely held assumption that such clinics see only chronic patients already in contact with the psychiatric services.

\section{Conclusion}

Existing walk-in clinics provide an accessible service to a widespread group of patients. A multidisciplinary team could deal more effectively with many referrals. The service provides an important route of entry into psychiatric care for many people, who might otherwise be delayed in their presentation. 


\section{Acknowledgements}

We thank Dr I. Pullen and the staff of the Medical Records Department for their help in this study.

\section{References}

Blaney, D. \& WeSt, A. (1987) Out of hours referrals to a General Psychiatric Hospital. Health Bulletin (Scotland), 45, 67-70.

Department of Health (1989) Caring for People: Community Care in the Next Decade and Beyond. London: HMSO.
Haw, C., Lanceley, C. \& Vickers, S. (1987) Patients at a psychiatric walk-in clinic-who, how, why and when? Bulletin of the Royal College of Psychiatrists, 11, 329332.

Johnstone, E. C., Crow, T. J., Johnson, A. L. \& MacMillan, J. F. (1986) The Northwick Park Study of first episodes of schizophrenia - presentation of the illness and problems relating to admission. British Journal of Psychiatry, 148, 115-119.

LiM, M. H. (1983). A psychiatric emergency clinic: a study of attendances over six months. British Journal of Psychiatry, 143, 460-466.

\title{
What would Adolf Meyer have thought of the neo-Kraepelinian approach?
}

\author{
D. B. Double, Lecturer in Psychiatry, University Department of Psychiatry, \\ Northern General Hospital, Sheffield S5 7AU
}

Because of the problem of diagnostic unreliability in psychiatry, there has been a trend over recent years to create explicit diagnostic criteria, such as the Research Diagnostic Criteria and DSM-III. This movement has been called neo-Kraepelinian (Klerman, 1978) as it promotes many of the ideas associated with the views of Kraepelin, regarded as the founder of modern psychiatry. It favours a biological approach and arose partially as a response to attacks on the 'medical model' in psychiatry.

Adolf Meyer (1866-1950) is remembered for his opposition to the preoccupation of the Kraepelinians with diagnosis. Although he accepted that there may be a place for classification, he argued that if diagnosis was meaningful, it was secondary to the assessment of the patient as a person. He was regarded as extremely influential in American psychiatry in the first half of this century, but his influence at the Phipps clinic, where he was the first director from when it opened in 1913, had faded by the mid-1950s when visited by Shepherd (1986) and, in general, little is now known about his contribution to psychiatry. A typical evaluation of his approach has been that it was "almost entirely sterile" (Slater \& Roth, 1969).

Whereas Meyer's writings are complex and turgid to read, there has been a clear-cut statement of the neo-Kraepelinian position. Klerman (1978) suggests there are nine assumptions which form the basis of the movement. Each of these propositions is listed below and examined hypothetically from Meyer's viewpoint. Because of Meyer's rejection of many of Kraepelin's views, it is reasonable to expect that he would have regarded the more modern approach as a similar escape from the uncertainties of psychiatry. It is probably because of his personal style that his contributions are now largely forgotten, perhaps in a similar way to Aubrey Lewis, who studied under Meyer, and whose legacy was negatively reviewed by Stengel (1968) among others. Such a tradition, though, should have more to offer to the current 